\title{
Calibration of the LSST instrumental and atmospheric photometric passbands
}

\author{
David L. Burke ${ }^{1}$, T. Axelrod ${ }^{2}$, Aurélien Barrau ${ }^{3}$, Sylvain Baumont ${ }^{3}$, Stéphane Blondin ${ }^{4}$, \\ Chuck Claver ${ }^{5}$, Alexia Gorecki ${ }^{3}$, Željko Ivezić ${ }^{6}$, Lynne Jones ${ }^{6}$, Victor Krabbendam ${ }^{5}$, Ming \\ Liang ${ }^{5}$, Abhijit Saha ${ }^{5}$, Allyn Smith ${ }^{7}$ R. Chris Smith ${ }^{8}$, Christopher W. Stubbs ${ }^{9}$, Christophe \\ Vescovi ${ }^{3}$, and the LSST Project Team ${ }^{10}$ \\ ${ }^{1}$ SLAC National Accelerator Laboratory, Menlo Park, CA, 94025 USA \\ ${ }^{2}$ Steward Observatory, University of Arizona, Tucson, AZ, 85718 USA \\ ${ }^{3}$ LPSC, CNRS-IN2P3, 53 rue des Martyrs, Grenoble, cedex 38026, France \\ ${ }^{4}$ CPPM, CNRS-IN2P3, 13288 Marseille cedex 09, France \\ ${ }^{5}$ National Optical Astronomy Observatory, Tucson, AZ, 85718 USA \\ ${ }^{6}$ Department of Astronomy, University of Washington, Seattle, WA, 98195, USA \\ ${ }^{7}$ Department of Physics and Astronomy, Austin Peay State University, Clarksville, TN, 37044 USA \\ ${ }^{8}$ Cerro Tololo Inter-American Observatory, Casilla 603, La Serena, Chile \\ ${ }^{9}$ Harvard Smithsonian Center for Astrophysics, Harvard University, Cambridge, MA, 02138 USA \\ ${ }^{10}$ LSST Corporation, 933 N. Cherry Avenue, Tucson AZ 85721
}

\begin{abstract}
The Large Synoptic Survey Telescope (LSST) will continuously image the entire sky visible from Cerro Pachon in northern Chile every 3-4 nights throughout the year. The LSST will provide data for a broad range of science investigations that require better than $1 \%$ photometric precision across the sky (repeatability and uniformity) and a similar accuracy of measured broadband color. The fast and persistent cadence of the LSST survey will significantly improve the temporal sampling rate with which celestial events and motions are tracked. To achieve these goals, and to optimally utilize the observing calendar, it will be necessary to obtain excellent photometric calibration of data taken over a wide range of observing conditions - even those not normally considered "photometric". To achieve this it will be necessary to routinely and accurately measure the full optical passband that includes the atmosphere as well as the instrumental telescope and camera system. The LSST mountain facility will include a new monochromatic dome illumination projector system to measure the detailed wavelength dependence of the instrumental passband for each channel in the system. The facility will also include an auxiliary spectroscopic telescope dedicated to measurement of atmospheric transparency at all locations in the sky during LSST observing. In this paper, we describe these systems and present laboratory and observational data that illustrate their performance.
\end{abstract}

Keywords: atmospheric effects, methods:observational, surveys, techniques:photometric

\section{Introduction}

The LSST telescope and camera system will produce a measure of the integral of the optical flux $F_{\nu}(\lambda)$ from a celestial source at the top of the atmosphere weighted by the observational bandpass, $S_{b}(x, y, a l t, a z, t, \lambda)$,

$$
C_{b}^{\text {meas }}=\Delta A \Delta T \int_{0}^{\infty} F_{\nu}(\lambda) S_{b}(x, y, \text { alt }, a z, t, \lambda) \lambda^{-1} d \lambda,
$$

where $b=($ ugrizy) denotes the broad-band filter used in the camera optics, $\Delta A$ is the area of the telescope input pupil, and $\Delta T$ is the duration of the exposure. The bandpass includes optical transmittance from the top of the atmosphere to the input pupil of the telescope, and the throughput of the optics, filters, and the sensors and electronics of the camera. The coordinates $(x, y)$ are those of the source image in the focal plane

\footnotetext{
${ }^{1}$ daveb@slac.stanford.edu
} 
of the camera, (alt,az) are altitude and azimuth of the telescope pointing vector, and $t$ is the time (and date) of the observation. The units of $C_{b}^{\text {meas }}$ are digitized counts (ADU), and the units of $S_{b}$ are ADU/photon. The weight $\lambda^{-1}$ reflects the fact that the CCDs used as sensors in the camera are photon-counting devices rather than calorimeters.

The LSST facility will include auxiliary subsystems to separately measure atmospheric transmittance and the instrumental response for each observation in the survey [Stubbs and Tonry 2006]. The system response will be measured in situ using a tunable monochromatic light source that will feed an array of projectors mounted in the LSST dome. The relative flux of light from these projectors will be monitored with a photodiode (or set of diodes) that has been precisely calibrated at the U.S. Institute of Standards (NIST).

The atmospheric transmittance will be measured using spectroscopic observations of bright isolated stars made with an auxiliary telescope placed near the LSST on Cerro Pachon. This telescope will repeatedly observe a small set of stars as they traverse the sky and backlight the atmosphere during the nights they are visible. Characteristic signatures of known atmospheric constituents in these spectra and a state-of-the-art physical atmospheric model will be used to extract the atmospheric mix present during LSST operations.

With the information that will be provided by the LSST auxiliary instrumentation, a measured bandpass for each observation made with the main telescope will be computed,

$$
S_{b}^{\text {meas }}(x, y, \text { alt }, a z, t, \lambda)=S_{b}^{s y s}(x, y, t, \lambda) \times S^{a t m}(a l t, a z, t, \lambda),
$$

where $S_{b}^{\text {sys }}$ (ADU/photon) is the measured instrumental system response, and $S_{b}^{\text {atm }}$ (dimensionless) is the measured atmospheric transmittance. A measured top-of-the-atmosphere in-band source magnitude will be defined in terms of measured quantities,

$$
m_{b}^{\text {meas }} \equiv-2.5 \log _{10}\left(\frac{C_{b}^{\text {meas }} / \Delta A \Delta T}{\int_{0}^{\infty} \lambda^{-1} F_{A B} S_{b}^{\text {meas }}(\lambda) d \lambda}\right) .
$$

The flux normalization $F_{A B}=3631 \mathrm{Jy}\left(1 \mathrm{Jansky}=10^{-26} \mathrm{~W} \mathrm{~Hz}^{-1} \mathrm{~m}^{-2}=10^{-23} \mathrm{erg} \mathrm{cm}^{-2} \mathrm{~s}^{-1} \mathrm{~Hz}^{-1}\right)$ is chosen for convenience. A measured normalized response function for each observation will also be defined,

$$
\phi_{b}^{\text {meas }}(\lambda) \equiv \frac{\lambda^{-1} S_{b}^{\text {meas }}(\lambda)}{\int_{0}^{\infty} \lambda^{-1} S_{b}^{\text {meas }}(\lambda) d \lambda} .
$$

The LSST photometry will be fully described by individual measurements of $C_{b}^{\text {meas }}$ and the corresponding $S_{b}^{\text {meas }}$. The survey will collect $\sim 10^{12}$ such $\left(C_{b}^{\text {meas }}, S_{b}^{\text {meas }}\right)$ pairs over a ten year period - one pair for each source detected in each image.

\subsection{The LSST Photometric System}

The LSST will use optical filters in the camera to define six photometric bands $b=$ ugrizy similar to the SDSS bands [Fukugita 1996]. Nominal instrumental bandpasses computed for these filters with the LSST design instrumental response are shown in Figure 1.

A set of LSST "standard" bandpasses $\phi_{b}^{\text {std }}(\lambda)$ will be defined as suitably weighted means or most probable $\phi_{b}^{\text {meas }}$ (Eq. 4) encountered during the commissioning (and perhaps first observing period) of the LSST survey. These bandpasses will define the LSST photometric "system", and will be used to compute LSST standard magnitudes for all sources and objects in the data catalogs. Observed magnitudes can be transformed to the standard system if the shape of the source SED is known, or at least approximately transformed if colors of the target are known. The difference between a magnitude computed with a measured $\phi_{b}^{\text {meas }}$ and that same magnitude transformed to $\phi_{b}^{s t d}$ is,

$$
m_{b}^{\text {std,meas }}-m_{b}^{\text {meas }} \equiv \Delta m_{b}^{s t d}=2.5 \log \left(\frac{\int_{0}^{\infty} f_{\nu}(\lambda) \phi_{b}^{\text {meas }}(\lambda) d \lambda}{\int_{0}^{\infty} f_{\nu}(\lambda) \phi_{b}^{\text {std }}(\lambda) d \lambda}\right) .
$$


The source SED $f_{\nu}(\lambda)$ is defined by

$$
F_{\nu}(\lambda)=F_{0} f_{\nu}(\lambda),
$$

with $f_{\nu}\left(\lambda_{b}\right)=1$ at some chosen reference wavelength. The value of $\lambda_{b}$ is entirely arbitrary, but a natural choice for this reference value is the mean wavelength of the normalized standard bandpass $\phi_{b}^{\text {std }}$.

\subsection{Photometric Data Products}

Equations 1 and 3 reveal a major limitation of broad-band photometry. Many SEDs vary substantially across the observational bandpass, so there is ambiguity in the meaning of an in-band magnitude computed with a constant, or flat, normaling flux $F_{A B}$. The proliferation of observational bandpasses noted above will also be a problem for many users of the LSST data catalogs. Use of standard magnitudes (Eq. 5) may eliviate this, but will only be valuable for sources whose SEDs or colors are reasonably well known.

In addition to the directly observed fluxes, magnitudes, and bandpasses defined in Sec. 1, the Source and Object Catalogs will include standard magnitudes (Eq. 5), and magnitudes computed with a number of appropriate SEDs (stellar, QSO, galaxy, SNe, etc.) for each entry. An interface will also be provided to compute magnitudes with user-supplied SEDs or target types. This will allow users to recompute, and perhaps fit, standard LSST magnitudes based on prior information about the SEDs of their targets.

\subsection{Requirements for LSST Photometric Calibration}

Science requirements have been established for the LSST survey that lead to minimum specifications for photometric precision and accuracy[Ivezic 2008]. Somewhat different design specifications are also given to guide the technical and engineering choices for the project, and stretch goals are given that would recognizably enhance the science output of the survey. These three sets of numbers are summarized in Table 1.

The first two rows give specifications on the stability of the calibration of repeated measurements of a single object and on the uniformity of the LSST flux scale at different celestial coordinates. The last two rows give specifications on the absolute calibration of measured colors and on the uncertainty in the definition of the overall LSST flux scale. These specifications are defined operationally for standard magnitudes of "bright isolated stars" for which the assignment of ADU counts can be made with little error and statistical counting errors are also small. This is done to (approximately) separate the task of assigning to individual sources the ADU counts from pixels in an image from the task of defining the physical meaning of (calibrating) an ADU count on a particular image. The calibration task is the one addressed by the process outlined in this paper.

Table 1: LSST Requirements for Stellar Photometry (10 ${ }^{-3}$ Magnitudes)

\begin{tabular}{lccc}
\hline Quantity & Design Spec & Minimum Spec & Stretch Goal \\
\hline Repeatability gri/uzy & $5 / 8$ & $8 / 12$ & $3 / 5$ \\
Spatial Uniformity & 10 & 15 & 5 \\
Color Accuracy & 5 & 10 & 3 \\
Physical Scale & 10 & 20 & 5 \\
\hline
\end{tabular}

\section{The LSST Calibration Procedure}

Two levels of LSST calibration will be carried out at differing cadences and with differing performance targets. A nightly data calibration based on the best available information will be used for quality assurance, generation of alerts to transcients, and for analyses that do not require the quality of performance specified in Table 1 . A 
more complete analysis will recalibrate the data accumulated by the survey at periodic "Data Release" dates, and it is this calibration that will be held to full specifications. This section describes the measured calibration data and their use in the Data Release calibration process.

LSST photometric calibration is separated into three parts that address different requirements:

- Relative calibration: normalization of internal (instrumental) measurements in a given bandpass relative to all other measurements made in the same bandpass.

- Absolute calibration of colors: determination of the five unique differences between flux normalizations of the six bands (color zero points).

- Absolute calibration of flux: definition of the overall physical scale of the LSST magnitude system.

This is made numerically explicit by writing the relation between measured and true standard magnitudes in a lowest-order decomposition,

$$
m_{b}^{\text {std,meas }}=m_{b}^{\text {std,true }}+\sigma+\delta_{b}(x, y, \alpha, \delta, t, \phi, S E D)+\Delta_{b} .
$$

The $\sigma$ are random photometric errors with net zero values after subtraction of known means, and the $\Delta_{b}$ are unknown offsets of the zero-points of the six photometric bands from the $F_{A B}$ normalization (Eq. 3). The $\delta_{b}$ are systematic errors of the internal zeropoint error around $\Delta_{b}$ that depend on position in the field of view $(x, y)$, position on the sky $(\alpha, \delta)$, date and time, the observational bandpass $(\phi)$, and the source SED. The average of $\delta_{b}$ over the survey is zero by construction, but a value must be determined for each source location in each image of the survey.

\subsection{Relative Calibration: Determination of $\delta_{b}$}

Precision relative calibration of LSST photometry will be accomplished by analysis of the repeated observations of bright isolated stars. The data products that are used in this process are shown in Figure 2. A Reference Catalog of calibration stars will be selected from the accumulated Object Catalog based on observed morphologies and estimated colors. These stars will have magnitudes $17<r<20$ to assure that measurements of their fluxes are not saturated or limited by photon counting statistics. They will be further selected to be free of overlap with other sources in order to minimize errors in assignment of ADU counts to their flux. There will be $\sim 10^{8}$ such stars in the Reference Catalog. They will be spaced by approximately one arcmin on the sky, with $\sim 200$ detected on each $14^{\prime} \times 14^{\prime}$ CCD (at the galactic pole). Each of these stars will eventually be observed 100-200 times in each of the six LSST filter bands. Calibration parameters extracted from analysis of the Reference Catalog will be used to calibrate all other sources detected on each image.

All images in the accumulated survey will first be reduced with the Instrument Signature Removal (ISR) and Detection pipelines that detect celestial sources and assign instrumental flux values $C_{b}^{\text {meas }}$ (Eq. 1) to each source. This process includes corrections for cross talk, electronic bias, and subtraction of backgrounds after flat-fielding. (See Sec.. 3.2.4 below.) The specific algorithm used by the ISR will be provided as part of the LSST calibration definition. The Calibration Pipeline (Figures 3 and 4) will use data taken with the auxiliary calibration subsystems, data from individual science images, and accumulated data in the Object Catalog to determine $S^{\text {atm }}$ and $S_{b}^{\text {sys }}$ for each image. (See Sec. 3 below.)

A global self-calibration procedure will subsequently be executed to minimize the dispersion of the $\delta_{b}$ of all observations of all reference stars. This process is based on techniques used in previous imaging surveys [Glazebrook 1994] [MacDonald 2004], and the specific implementation used by LSST will be based on the "Übercal" procedure developed for SDSS [Padmanabhan 2008]. "Calibration patches" of a certain size will be defined on the camera focal plane. The optimum size for these patches will be determined by simulation and analysis of precursor data, but will naturally be similar to a CCD footprint. So there will be $\sim 200-400$ calibration patches per image, and each star $i$ on each image $j$ will be assigned to a patch $p$. The LSST survey is specifically designed to "dither" pointings from epoch to epoch to control systematic errors, so stars will fall 
on different patches on different epochs across the sky. The measured magnitudes of reference stars will be transformed (Eq. 5) to the LSST standard bandpass using the accumulated estimates of the colors of each star and the corresponding measured observational bandpasses.

The Calibration Pipeline will determine relative errors $\delta_{b}(p, j)$ in the photometric zero-point for each patch $p$ on each image $j$ of the accumulated survey by minimizing,

$$
\chi^{2}=\sum_{(i, j)} \frac{\left(m_{b}^{\text {std,meas }}(i, j)-\left(m_{b}^{\text {std,fit }}(i)+\delta_{b}(p, j)\right)\right)^{2}}{\left(\delta m_{b}^{\text {std,meas }}(i, j)\right)^{2}}
$$

where the summation is over all stars $i$ in all images $j$. Here $\delta m_{b}^{\text {std,meas }}$ is the best estimate of the error in the measured standard magnitude (including uncertainty in the SED), and the fitted parameters are the best estimates of the true in-band magnitudes of each reference star $m_{b}^{\text {std,fit }}$ and the systematic errors in the zero points $\delta_{b}(p, j)$. These $\delta_{b}(p, j)$ will be used to correct the photometry for all other sources in patch $p$ on image $j$. There will be $\sim 10^{8}$ values of $m_{b}^{\text {std,fit }}$ and $\sim 2 \times 10^{8} \delta_{b}(p, j)$ per band to be determined in the accumulated survey, and there will be $\sim 10^{10}$ measurements of main sequence stars per band to constrain the fitted parameters. It remains to determine what computational technique works best to minimize this function.

In the formulation of Eq. 8 the patch index represents a spatial coordinate and the image index represents a temporal coordinate. It will be possible to analyze the fitted $\delta_{b}(p, j)$ to test for systematic errors in the initial calibration data products. For example, the distribution of $\delta_{b}$ versus celestial coordinates $(\alpha, \delta)($ Eq. 7$)$ can be used to test for residual errors in the uniformity of the atmospheric corrections. (See Sec. 2.2 below for further discussion of analyses that will be done to test for systematics in the calibration process.)

\subsection{Absolute Calibration: Determination of $\Delta_{b}$}

The global relative calibration minimizes the dispersion of measurement errors, but it does not address and in fact may introduce, systematic offsets $\Delta_{b}$ of the calibration scale from the $F_{A B}$ normalization defined in Eq. 3 . These six numbers can be expressed in terms of a single fiducial band, say $r$,

$$
\Delta_{b}=\Delta_{r}+\Delta_{b r}
$$

Any band could be chosen for the fiducial, but with this distinction, the design requirement for $\Delta_{b r}$ is 5 millimag and is 10 millimag for $\Delta_{r}$.

The five $\Delta_{b r}$ will be determined from the measured flux from one or more celestial sources whose physics and chemistry are believed to be well understood. In principle, a single object with known colors would be sufficient. Several WDs in the Northern hemisphere have been very precisely calibrated with HST STIS measurements [Bohlin and Gilliland 2004]. It may be possible to obtain similar HST measurements of one or more targets for use by surveys in the Southern hemisphere. But a broader range of celestial sources will be used to constrain the LSST color scale and to allow evaluation of possible systematic effects on science analyses.

Hot hydrogen (DA) and helium (DB) white dwarf stars have simple atmospheres that are reasonably well understood (model colors are currently reliable to about $0.01 \mathrm{mag}$ ). It is estimated that there will be $\sim 100$ (10) DA (DB) WD stars with $r<24$ in each LSST image at the South Galactic Pole. Catalogs of WD stars visible from Cerro Pachon have been constructed [Bergeron 1992] [Eisenstein 2006], and a "white dwarf calibration system" has been developed [Holberg and Bergeron 2006]. The locus of main sequence stars in color-color space is also reasonably well understood, and has been used to calibrate photometry with success in previous surveys [MacDonald 2004] [Ivezic 2007]. Initial rough calibrations might be done entirely with the main sequence locus, and ultimately this can be used to determine the effects of galactic extinction on calibration standards [Sale 2009] [High 2009]. 
At least one external flux standard will be required to determine $\Delta_{r}$ (one number for the whole survey!). While one celestial standard would be formally sufficient, chosing a number of such standards would provide a powerful test for $\Delta_{r}$. Identification of such a standard, or set of standards, has not yet been done.

\section{$3 \quad$ LSST Calibration Subsystems and Processes}

Flow-down of calibration performance requirements to the high-level data products used in the LSST photometric calibration is given in Table 2. These error budgets are assigned to assure that the calibration will achieve the Design requirements. They are made with the assumption that the input measurements contribute independently to the overall calibration error. However, it is realized that the global self-calibration procedure effectively couples these terms to minimize the dispersion of relative errors. The LSST strategy is to set specifications that will meet Design requirements without the benefit of improvement from the self-calibration, and recognize that this may allow LSST calibrations to meet Stretch goals. This approach also provides a measure of assurance that the Design requirements will be met.

Table 2: Flow Down of LSST Design Specifications (10 $10^{-3}$ Magnitudes)

\begin{tabular}{lccc}
\hline Design Specification & $\begin{array}{c}\text { Repeatability } \\
\text { gri } / u z y\end{array}$ & Uniformity & Color Accuracy \\
\hline Overall & $5 / 8$ & 10 & 5 \\
Instrumental Calibration $\left(S_{b}^{\text {sys }}\right)$ & $3 / 5$ & 5 & 3 \\
Atmospheric Characterization $\left(S^{\text {atm }}\right)$ & $3 / 5$ & 5 & 3 \\
Reference Catalog $\left(m_{b}^{\text {std,meas }}\right)$ & $3 / 4$ & 7 & 3 \\
\hline
\end{tabular}

\subsection{The Reference Catalog}

The last row in Table 2 is the accuracy with which standard magnitudes of stars in the Reference Catalog must be extracted from the measured data - these are essentially "nuisance" parameters in the calibration fit. This includes both errors in the extraction of ADU counts from images that contain these stars and the effect of uncertainty in the transformation of the measured instrumental magnitudes to corresponding standard magnitudes. The full SEDs of the reference stars will not generally be known, so an approximate color transformation must be used to make this transformation. The reference stars will be chosen and their colors sufficiently well measured to minimize the resulting error.

Stars will be chosen for the LSST Reference Catalog based on photometric measurements made initially by precursor surveys. The SDSS Equitorial Stripe 82 catalog of $300 \mathrm{deg}^{2}$ is already available with photometric errors of $1 \%$ or better. The SkyMapper survey [Keller 2007] will cover the same $2 \pi$ of the Southern hemisphere planned for LSST. The deeper DES survey that will cover a portion of the LSST survey area will be carried out in this time period, and the PanSTARRS survey will also include fields in the Southern Hemisphere. The photometry of these precursor surveys will be adequate for the initial references for LSST, and measurements with the LSST itself will eventually replace these initial data.

\subsection{Measurement of Instrumental System Response $S_{b}^{\text {sys }}$}

Shown in Figure 3 is a schematic of the process used to measure the LSST instrumental system response $S_{b}^{\text {sys }}$. This section describes this process and the major subsystems that provide data for it. 


\subsubsection{The Monochromatic Dome Projector System}

The function of the Monochromatic Dome Projector system is to provide a well-controlled source of light for measurement of the relative throughput $S_{b}^{s y s}(x, y, t, \lambda)$ of the full LSST instrumental system. This includes the reflectivity of the mirrors, transmission of the refractive optics and filters, the quantum efficiency of the sensors in the camera, and the gain and linearity of the sensor read-out electronics. Spatial, temporal, and chromatic variations of the throughput will be determined up to a single overall normalization for the accumulated LSST survey.

A critical functional requirement is that the screen provide a smooth illumination uniform over spatial scales smaller than the sizes of the patches used in the global self-calibration procedure. The flux of light produced by the screen must fill the LSST aperture, but the intensity of light need only be uniform to $10 \%$ or so across the telescope pupil. Scattering of light from outside the telescope beam into the view of the camera must be controlled, so collimation schemes are being investigated to limit the light emitted at wide angles from the screen.

The present design is to use an array of projectors mounted in the dome of the LSST enclosure that can be illuminated with either broadband (e.g. quartz lamp) or a tunable monochromatic light source. These "flat-field" projectors are designed to fill the LSST etendue with uniform illumination, and also to limit the extent of stray light emitted outside the design acceptance of the system. A set of precision diodes will be used to normalize the photon flux integrated during flat-field exposures. These photodiodes, together with their read-out electronics, will be calibrated at the U.S. National Institute of Standards (NIST) to $\sim 0.1 \%$ relative accuracy across wavelength from $450 \mathrm{~nm}$ to $950 \mathrm{~nm}$. The response of these diodes varies smoothly across this range of wavelength (Figure 5). Adjustment of the wavelength of the light source can be as fine as one nanometer, and will allow precise monitoring of the shape of the bandpasses of the instrumental system during the course of the survey. A conceptual set-up at CTIO has been used to demonstrate the feasibility of this technique (Figure 6) [Stubbs 2006].

It is anticipated that the shapes of the bandpasses will vary only slowly, so detailed measurement will need be done once per month or so. But build up of dust on the surfaces of the optics will occur more rapidly. Daily broadband (e.g. quartz) flat-field measurements will provide corrections for the "gray" absorption by dust particles whose dimensions are large compared to the wavelengths of light in the LSST bandpasses, and whose shadows will be out of focus at the focal plane. Full measurement of the bandpasses will be required after cleaning of mirror surfaces or other known changes in the throughput of the system.

\subsubsection{Dome Flat Illumination Correction}

It is anticipated that, despite careful design, the illumination pattern from the dome projector system will not be uniform over spatial scales comparable to the size of the focal plane. Estimates ${ }^{2}$ of the flux of "stray" light within the dome housing indicate that $\sim 1-2 \%$ of the light that reaches the camera focal plane may not originate from within the LSST etendue. And the telescope mirror optics overfills the camera aperture, so there will be light scattered within the camera dewar. It is also estimated that $1-2 \%$ of the light that is within the etendue will have undergone multiple reflections within the camera refractive optics, so will be out of focus when it reaches the focal plane and appear as an accumulation of ghost light. There are also well-known projection effects that must be taken into account to fully translate a dome-flat to a true celestial flat-field.

Subtraction of backgrounds in science images by the ISR pipeline will substantially reduce the effects of stray and scattered light on ADU counts assigned to celestial sources. But no background subtraction is possible for dome exposures since they fill the focal plane with nearly uniform light. An illumination correction must be determined and applied to the dome screen images before they are used to process science exposures. Dedicated rastored scans of dense star fields coordinated with dome screen exposures will be used to measure the differ-

\footnotetext{
${ }^{2}$ Calculations done by Photon Engineering Inc., Tucson, AZ.
} 
ence between the illumination of the focal plane by celestrial sources and that produced by the dome screen. This technique has been successfully used in previous surveys [Manfroid 1996] [Magnier and Cuillandre 2004].

\subsubsection{Calibration of the LSST Imaging Camera}

A Camera Calibration Optical Bench (CCOB) will provide a carefully controlled source of light to illuminate the focal plane of the LSST camera while it is dismounted from the telescope. The CCOB will be used to fully characterize the spatial and chromatic response, $S_{b}^{F P A}(x, y, \theta, \phi, \lambda)$, of the camera sensors and electronics under a range of operating conditions. This can be done in isolation of the refractive optics (except the final cryostat window) to minimize ghosting effects. And it can be done through the full refractive optics to characterize ghosting patterns from the camera optics and filters.

A pencil beam will be scanned across the focal plane in steps small compared to the central spot diameter (a few centimeters). The spot intensity profile is then measured by individual pixels, and the response of each pixel relative to a suitable mean can simultaneously be extracted from the data. The CCOB will allow the trajectory of the incident light to be varied to allow study of the response of pixels over the range of angles in the focused LSST optical beam. The light source will provide both narrow and broad-band beams, and the integrated flux of the light during exposures will be monitored with a precision photodiode calibrated to those used to monitor dome exposures. Thermal time constants of the system, and changes in response of the electronic properties of the focal plane with temperature, will be determined.

The CCOB will be used to calibrate (ZEMAX) model calculations of ghosting patterns to support determination of the dome illumination correction. The intensity and positions of ghost patterns in the focal plane can be used to extract the reflectivities and alignments of the optical surfaces of the camera optics. And their shapes will be sufficiently well predicted to use in examination and fits to data taken with the Dome Projector System (Section 3.2.1 above).

\subsubsection{Integration of Instrumental Calibration Data: Flat-Fielding}

A schematic of the integration of the data from the various instrumental calibration subsystems with the data taken with the main telescope is shown in Figure 3. A summary of calibration operations is also given in Section 4. The Calibration Pipeline will be responsible for most of the steps in this schematic, while the ISR pipeline will apply the flat-field correction, $S_{b}^{s y s}$, to images taken with the main telescope.

The paradigm displayed in Fig. 3 incorporates a "Template Flat" that embodies most, if not all, of what is known about the instrumental system at various fixed points in the data stream. This Template will be synthesized from the full stack of exposures made with the Monochromatic Dome Projector system and the measured Illumination Correction. There are several possible wavelength weighting schemes that will be used to arrive at flat fields that best support science goals. This will result in multiple entries in the data catalogs as appropriate. The "DIA Flat", or difference flat, tracks changes in the system between updates of the monthly Dome stacks. It will be based on the nightly broadband flats and mini-monochromatic scans. Instrumental meta-data, such as the instantaneous temperature profile across the focal plane, may be used to make small corrections to the flat for each image. The Nightly calibration will, of course, take place in real time and have only the benefit of prior experience. But the Data Release calibration algorithms will analyse the accumulated survey data, and may benefit from smoothing or fitting data gathered between points of major change.

\subsection{Measurement of Atmospheric Transmittance $S^{\text {atm }}$}

Shown in Figure 4 is a schematic of the process used to determine the atmospheric transmittance $S^{a t m}$ for each source in each LSST image. This section describes this process and the major subsystems that provide data for it.

The LSST calibration strategy is to measure the full spatial and temporal variation in atmospheric extinction throughout each night independently of operations of the main survey telescope. This will be done by 
taking spectra of a small set of probe stars with a dedicated auxiliary telescope and combining this data with the analysis of the LSST images themselves. The probe stars will be spaced across the sky to fully cover the area surveyed by the LSST main telescope, and they will be observed repeatedly as they traverse the sky each night they are visible. This will allow the atmospheric mix present along any line of sight at any time to be interpolated from the measured data. The probe stars will be observed many times during the ten-year LSST survey, so the SED of each star can be bootstrapped from the data. The instrumental response of the spectrograph can also be bootstrapped from the data and monitored with well-known photometric standards included in the catalog of probe stars. The calibration will use state-of-the-art atmospheric optical transport models and readily-available codes to accurately compute the signatures of all significant atmospheric components in these spectra. This includes molecular scattering and absorption from oxygen, nitrogen, ozone, water vapor, and trace elements. Scattering by aerosols is also included with both optical depth and spectral index taken as free parameters fit to the data. A "gray" component is included to account for water droplets and ice in clouds that are large compared to the wavelength of visible light and therefore cast wavelength-independent shadows on the field of view of the telescope and camera.

An observational campaign was carried out using the SMARTS CT1.5/CSPEC on Cerro Tololo near the planned LSST site to test the feasibility of this technique and to set the design parameters for the calibration subsystem. These studies have shown that it is possible to characterize the chemical mix of the atmosphere sufficiently well to meet performance requirements for stability and uniformity of photometric precision. Shown in Figures 7, 8, and 9 are plots that summarize the results of these studies[Burke 2010]. The observations made during this study were separated into a calibration set used to extract the atmospheric parameters, and a second "blind" set that were not used in the calibration and only examined after the calibration was completed. The figures show results of applying the calibration to the blind test observations. In each case, measurements of calibrated magnitudes or colors derived for individual observations are compared to the corresponding average quantity for the set of observations from the same star. If we assume that no correction can be made for "gray" extinction, then the residual (Figure 7) reflects the variation seen in the cloud cover as it passed over the site. Clear correlations of cloud cover in time are observed in the data, and some improvement can be gained by interpolating between calibration observations to other points in space and time. There is a set of observations for which this makes a substantial improvement. But the spatial structure of the cloud cover is such that a correction computed from a spectrum taken at one time or location can not be used to accurately correct an observation taken at different times or elsewhere.

To examine the completeness of our model of atmospheric extinction we first use values of "gray" that are extracted from fits to the blind observations of the probe stars. These residuals are shown in Figure 8. The displayed rms variances can be compared with the precision of the data estimated from the statistical counts in the spectra to be 0.002 in $g r i, 0.003$ in $z$, and 0.005 in $y$. It is evident that all other calibration factors transfer accurately to the blind observations in the griz bands, with some degradation in the $y$ band. Residual contributions due to errors in the atmospheric model, the fitting procedure, and sampling limitations are seen to be most significant in the $y$-band where they are comparable to the statistical error. As a further test we look at colors computed as differences of magnitudes derived from the same observed spectrum; these will be independent of true "gray" extinction. The dispersion of colors computed from blind observations are shown in Figure 9. These color dispersions are somewhat smaller than would be expected if the residual errors in separate bands are independent. This is consistent with there being remaining errors in the atmospheric model that introduce correlated errors in the individual band magnitudes. But the consistency of the dispersion of the corrected magnitudes and the color magnitudes leads to the important conclusion that our model accounts for all contributions to extinction larger than $\sim 3$ millimagnitudes in these data.

While the results of our observing tests demonstrate the ability to correct for scattering and absorption for molecular and aerosol constituents of the atmosphere, they also show that cloud cover changes too rapidly on too fine a spatial scale to be tracked with this technique. The LSST survey demands that photometric specifications be met in conditions that include significant cloud cover - i.e. during times that are not "photometric". The strategy for correcting measured fluxes for cloud cover utilizes the calibration reference stars seen in each image and an auxiliary IR camera that is co-bore sighted with the LSST. Coordinated runs were 
begun in 2008 with the CT $1.5 \mathrm{~m} / \mathrm{CSPEC}$ and the CT $0.9 \mathrm{~m} / \mathrm{CFIM}$ imager to begin to learn how to carry out this process. Spectra of probe stars were acquired while imaging fields with densities of stars similar to those expected in LSST single images. For example, a field in the southern outskirts of NGC5139 was followed for three nights over a wide range of airmass. The small aperture of the imaging telescope required longer exposure times than the 15 seconds planned for LSST, so spatial structures in cloud formations as small as will be encountered in LSST images were not resolved in these studies. Images were taken with exposure times of 30 seconds to several minutes to obtain a gauge of this effect. Magnitudes of stars in each image were first corrected for wavelength-dependent extinction, and the residual pattern of "gray" extinction then fitted across the $13^{\prime} \times 13^{\prime}$ FOV of the CT0.9m/CFIM with second-order spatial polynomials. The resulting magnitude dispersions (Figure 10) are found to be limited by photon statistics and instrumental errors. But at the bright end of the spectrum of stars it is seen that these initial studies have reached precisions of $1 \%$ or better through 2 magnitudes of cloud extinction or more.

\subsubsection{Auxiliary Calibration Telescope}

The function of the Auxiliary Telescope (AT) is to measure spectra of stars with sufficiently fine sampling in spatial coordinates and time to determine the wavelength dependence of optical transmission of light from the top of the atmosphere to the input pupil of the telescope for each LSST image. The LSST design includes a $1.2 \mathrm{~m}$ auxiliary calibration telescope located on Cerro Pachon near the LSST. Recognition of the signatures of atmospheric components is done with relatively modest spectral resolution $(R \approx 400)$, but it is important that the full spectral range $(330 \mathrm{~nm}$ to $1100 \mathrm{~nm})$ be obtained in a single exposure. To keep up with changes in the atmospheric mix (particularly water vapor), this subsystem must be able to slew and capture spectra of relatively bright stars $(r<12)$ at a reasonable pace (every 5 minutes).

\subsubsection{Thermal IR Cloud Camera}

A camera operating at IR wavelengths will be installed and co-bore sighted with the LSST main telescope to provide imaging of the thermal contrast between clouds and the night background sky. Narrow band filters will select a window (e.g. $9.2 \mu \mathrm{m})$ in the atmospheric transmission of light to provide greatest sensitivity to thermal emission from clouds. This camera will image the LSST field of view with resolution of 0.25 arcmin or less, and will provide sensitivity to clouds that produce gradients of more than a few millimags of extinction over this spatial scale. Cameras that can meet this goal are commercially available ${ }^{3}$.

\subsubsection{Integration of Atmospheric Data: Extinction Correction}

Shown in Fig. 4 is a flowchart of the data processing required to compute the atmospheric transmittance $S^{a t m}(a l t, a z, t, \lambda)$ from spectra of probe stars taken with the Auxiliary Telescope and the image data from the LSST main telescope and thermal IR Cloud Camera. Spectra from the Auxiliary Telescope are reduced using well-known software packages (e.g. IRAF), and normalized with the spectra of spectrophotometric standard stars that are included in the probe star catalog. This normalization includes correction for the efficiency of the spectrograph, but not correction for atmospheric extinction. An iterative fitting procedure is then used to extract parameters (effective temperature, gravities, and luminosities) for each of the probe stars (once per Data Release), nightly corrections for the spectrograph efficiency, and coefficients that define the mix of constituents in the atmosphere. The Auxiliary Telescope is designed to take spectra fast enough that the atmospheric extinction for each LSST pointing can be interpolated from the data from the probe stars.

The mix extracted from fits to the Auxiliary Telescope spectra includes all known constituents in the atmosphere except the large water droplets and ice crystals that comprise clouds. These particles are physically large compared to the wavelength of visible light, and so cast a shadow that is independent of wavelength across the LSST bandpasses. The "gray" opacity and spatial structure of clouds within each LSST image will be extracted from smoothing fits to the observed magnitudes of the Reference Stars (Figure 2). Images from

\footnotetext{
${ }^{3}$ See, for example, http://www.flir.com.
} 
the Thermal IR Camera will be normalized to these magnitudes and used to extrapolate the resolution of the cloud structure to smaller spatial scales.

\section{Calibration Operations}

A summary of expected calibration data is given in Table 3. The data is separated into those taken on a daily or nightly basis, and those to be taken over a period of time of approximately one month. The LSST calibration design obviates the need to use observing time with the main telescope for calibration tasks. The dome screen can be used during non-observing periods to fully characterize the instrumental response of the telescope and detector. Routine calibration processes will utilize the usual techniques of making bias and dark exposures to determine instrumental offsets and backgrounds, and sky flats constructed from science images will be used to check for systematic errors in flat-fielding the instrument.

The optical bandpasses of the telescope and camera systems can be monitored with miniscans of a few wavelengths for each filter that is used on a given night of observing. Particular care will be taken to monitor the edges of each bandpass. The broad-band flat-field response will be measured at the start and end of each observing night. More complete examination of the full instrument response will require several hours; these measurements will be accumulated over approximately monthly periods during periods of poor weather.

Table 3: Summary of Calibration Operations

\begin{tabular}{cll}
\hline $\begin{array}{c}\text { Activity } \\
\text { Subactivity }\end{array}$ & Purpose & Cadence \\
\hline Main Telescope Calibration Images & & \\
Bias Frames & Bias subtraction & Nightly \\
Darks & Instrument QA & Nightly \\
Broadband Dome Flats & Flat field/Instrument QA & Nightly \\
Monochromatic Mini Scans & Flat field/Bandpass QA & Nightly \\
Monochromatic Full Scans & Bandpass determination & Monthly \\
Photon Transfer Curves & System linearity & Monthly \\
Main Telescope Sky Images & & \\
Rastored Standard Fields & Flat field illumination & Monthly \\
Co-Bore Sighted Cloud Camera & & \\
Blackbody Images & IR calibration & Nightly \\
Sky Images & Cloud extinction & LSST cadence \\
Calibration Auxiliary Telescope & 2-D image reduction & Nightly \\
Bias and Dome Flats & Spectrograph calibration & AuxScope cadence \\
He-Ne and Quartz Calibration & Atmospheric extinction & AuxScope cadence \\
Spectra & &
\end{tabular}

\section{Acknowledgments}

This work has been done as part of the design and development activity of the Large Synoptic Survey Telescope (LSST). LSST is a public-private partnership. Design and development activity is supported by in part the National Science Foundation under Scientific Program Order No. 9 (AST-0551161) and Scientific Program Order No. 1 (AST-0244680) through Cooperative Agreement AST-0132798. Portions of this work are supported by the Department of Energy under contract DE-AC02-76SF00515 with the SLAC National Accelerator Center, contract DE-AC02-98CH10886 with Brookhaven National Laboratory, and contract DE-AC52-07NA27344 
with Lawrence Livermore National Laboratory. Additional funding comes from private donations, grants to

universities, and in-kind support at Department of Energy laboratories and other LSSTC Institutional Members.

\section{References}

[Bergeron 1992] Bergeron, P., Saffer, R.A., and Liebert, J., AJ 394228 (1992)

[Bohlin and Gilliland 2004] Bohlin and Gilliland AJ, 128, 3053 (2004)

[Burke 2010] Burke, D., et al., submitted to ApJ (2010)

[Eisenstein 2006] Eisenstein, D.J. et al., AJ Suppl. 167, 40 (2006)

[Fukugita 1996] Fukugita, M. et al., AJ, 111, 1748 (1996)

[Glazebrook 1994] Glazebrook K.,Peacock J.A., Collins, and Miller, L., MNRAS 266, 65 (1994)

[High 2009] High, F.W., et al., astro-ph/0903.5302, submitted to AJ (2009)

[Holberg and Bergeron 2006] Holberg, J. B. and Bergeron, P, AJ 132, 1221 (2006)

[Ivezic 2007] Ivezic, Z, et al., AJ 134, 973 (2007)

[Ivezic 2008] Ivezic, Z., et al., astro-ph/0805.2366 (2008)

[Keller 2007] Keller, S. C., et al., astro-ph/0702511 (2007)

[MacDonald 2004] MacDonald, E.C., et al., MNRAS 352, 1255 (2004)

[Magnier and Cuillandre 2004] Magnier, E.A. and Cuillandre, J.-C., PASP 116, 449 (2004)

[Manfroid 1996] Manfroid, J., A\&AS 118, 391 (1996)

[Oke and Gunn 1983] Oke, J. B. and Gunn, J. L. ApJ 266, 713 (1983)

[Padmanabhan 2008] Padmanbhan, N, et al., ApJ 674, 1217(2008)

[Sale 2009] Sale, S.E., et al., MNRAS, 392, 497 (2009)

[Stubbs and Tonry 2006] Stubbs, Christopher W. and Tonry, John L., ApJ, 646, 1436 (2006)

[Stubbs 2006] Stubbs, C, et al., AAS 207th Abstract 180.09 (2006); see also astro-ph/1003.3465 (2010) 


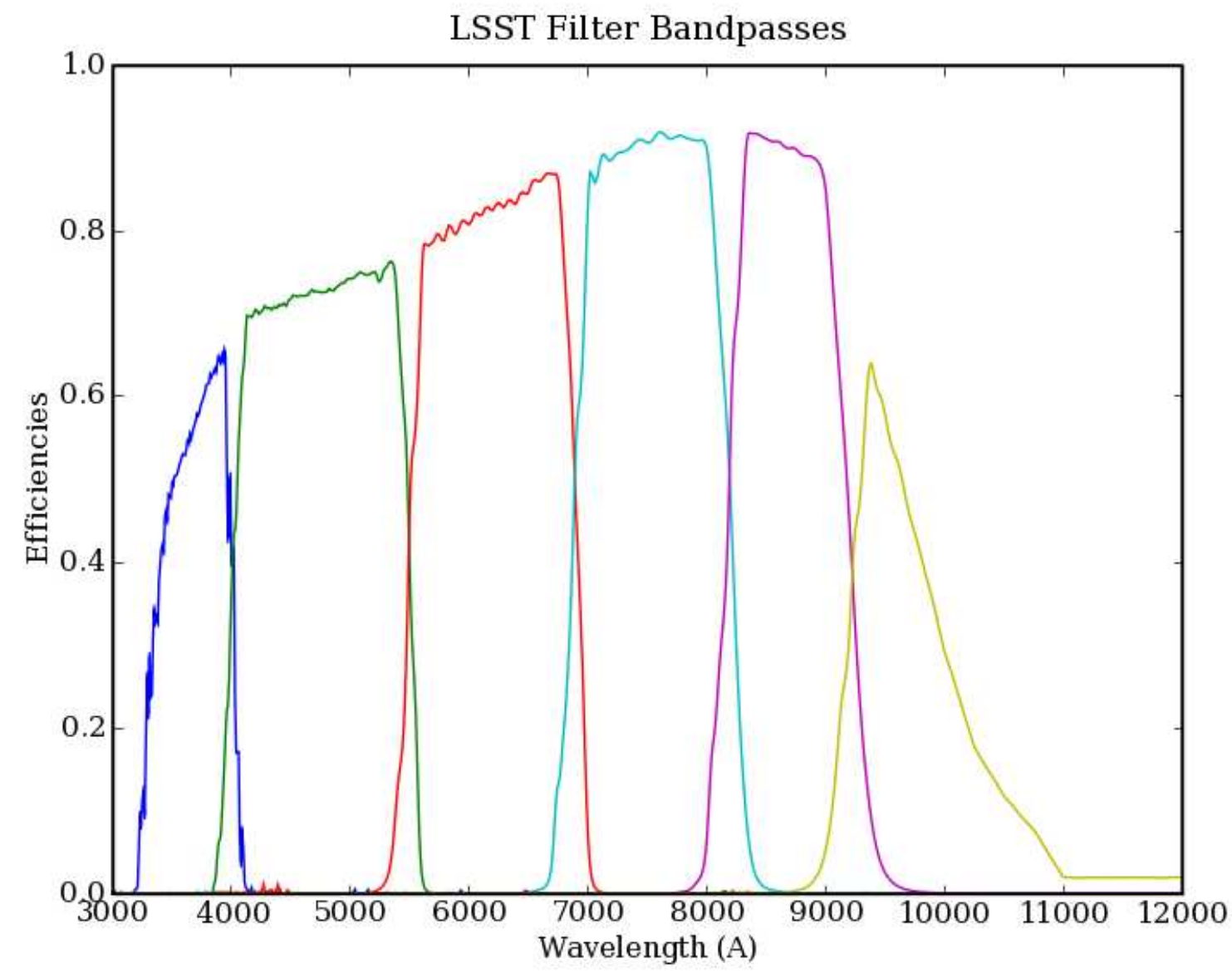

Figure 1: Nominal LSST instrumental ugrizy bandpasses. The curves are computed for the design system response of the telescope, camera, and filters. 


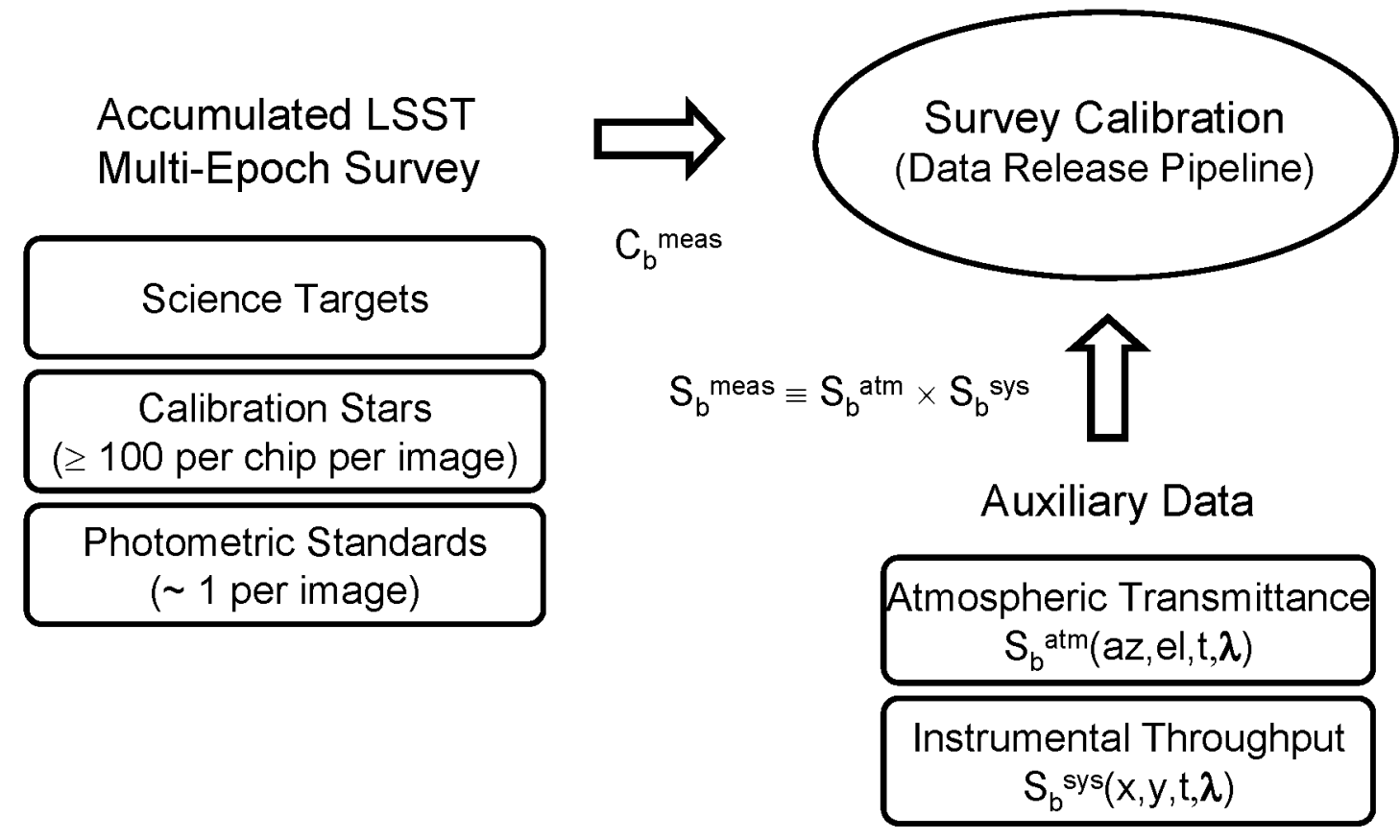

Figure 2: Measured data products used in the LSST Data Release calibration process. The quantities are defined in the text. 


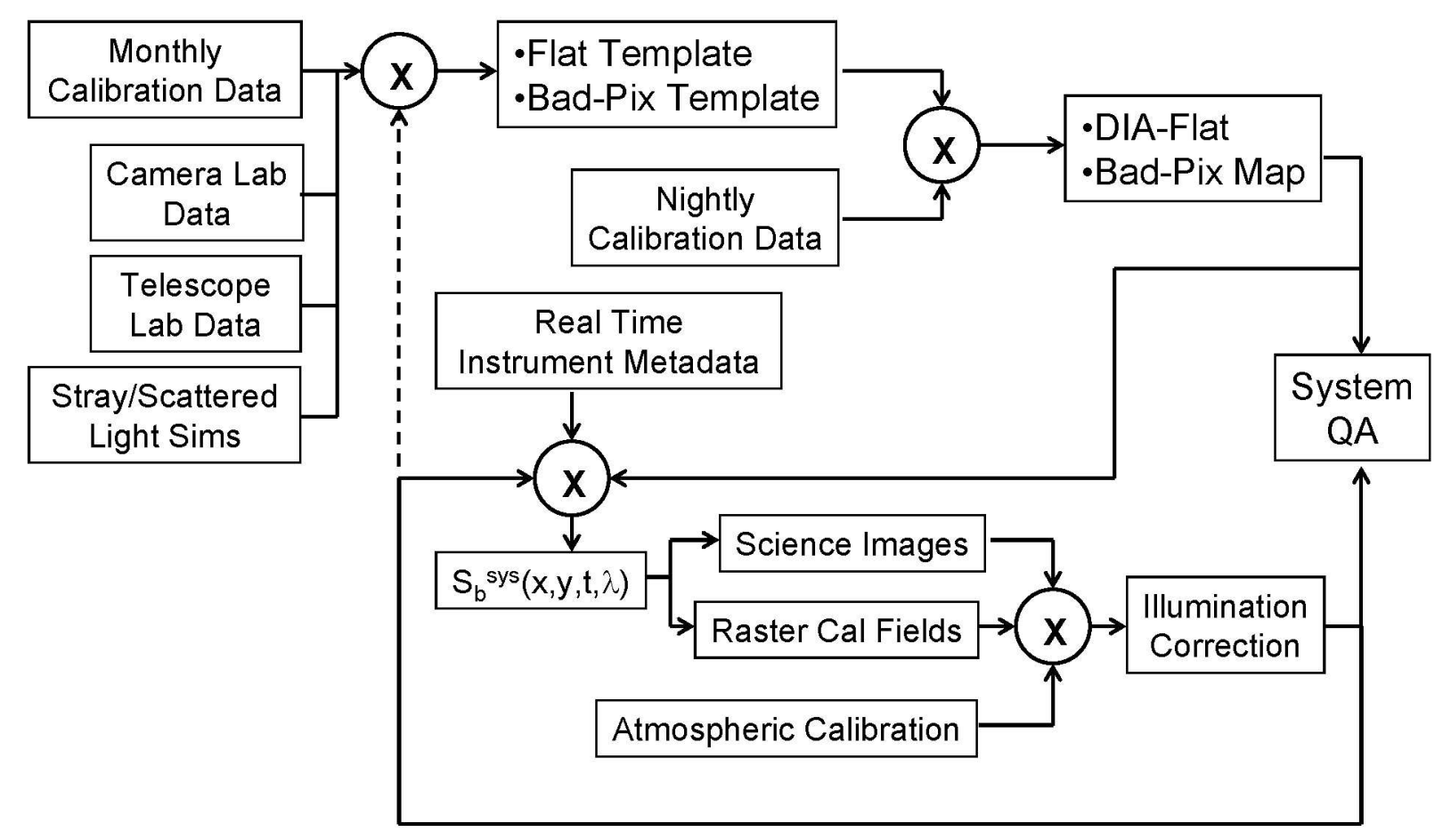

Figure 3: Schematic of the data flow used in the measurement of the instrumental system throughput. This schematic can be viewed as a process occuring in real time (e.g. the Nightly calibration), or as an organization diagram for analysis of the data accumulated by the survey (e.g. the Data Release calibration). Processes that include quality cuts and high and low temporal and spatial filtering of the calibration data are indicated by the encircled "X" marks. 


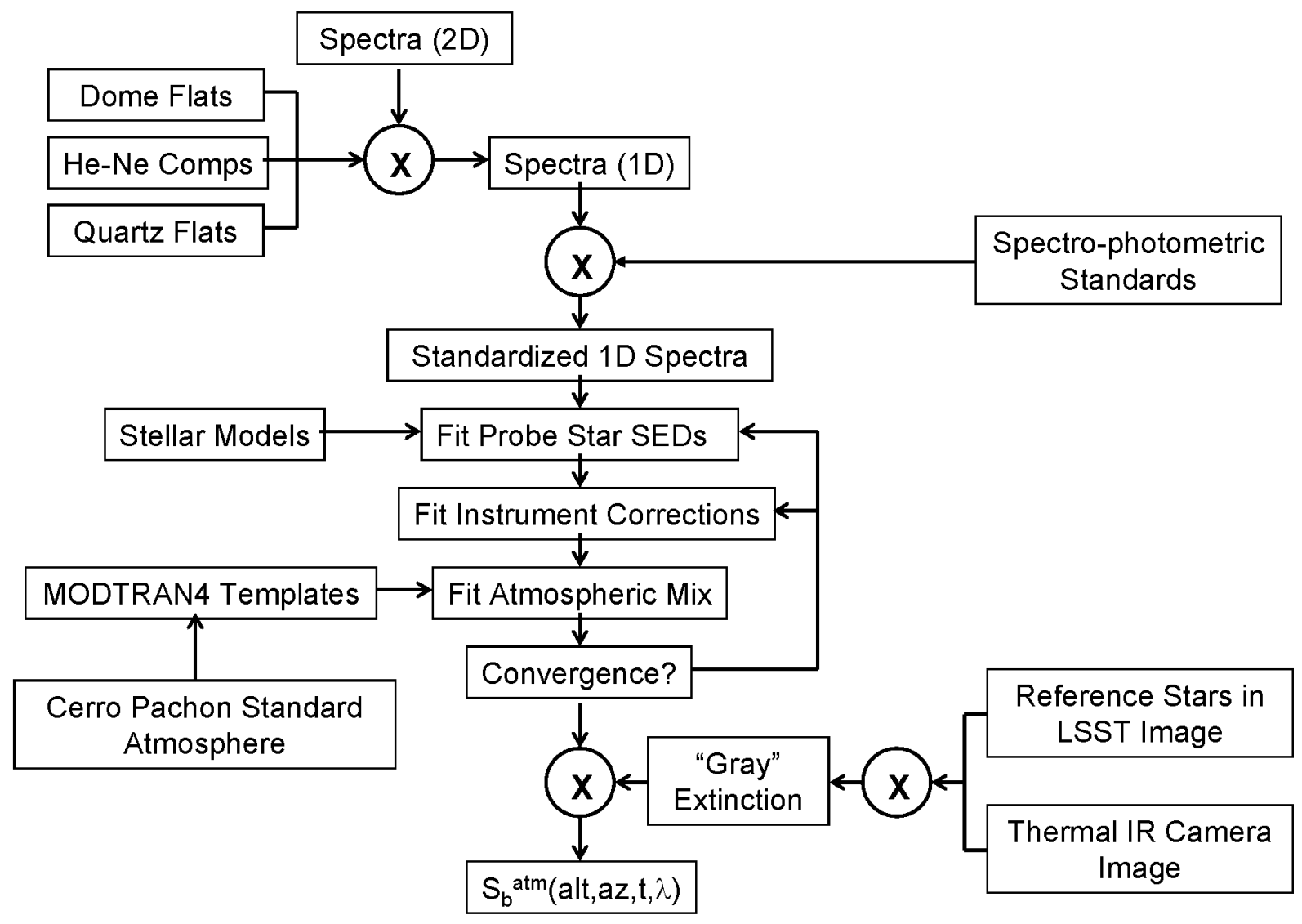

Figure 4: Schematic of the data flow used in the measurement of atmospheric extinction. Nightly corrections for extinction can be extracted from non-iterative fits to individual probe star spectra. Reprocessing of the accumulated data with the fully iterative fitting procedure will be executed for each Data Release. 


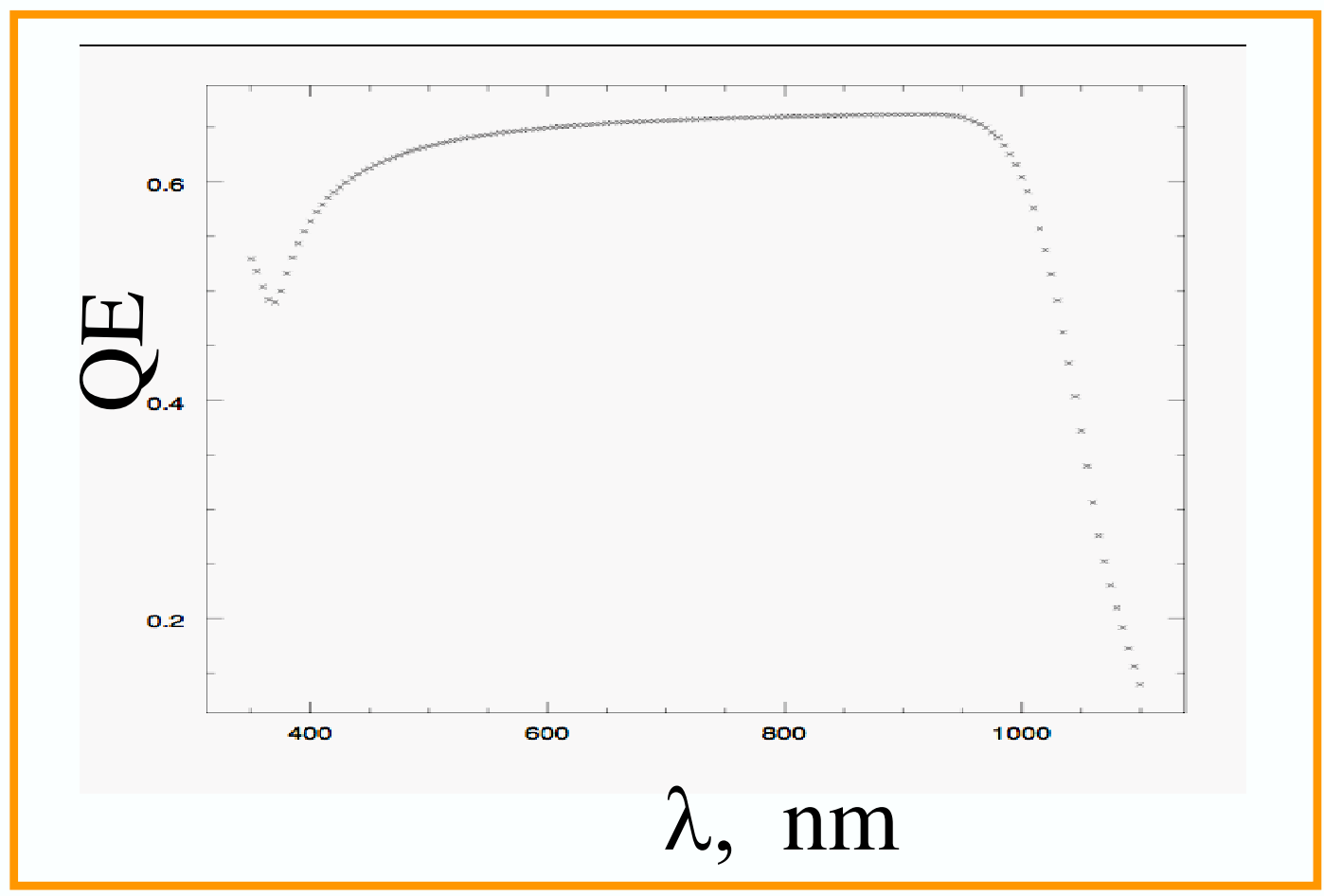

Figure 5: Response function of a precision diode calibrated at the U.S. National Institute for Standards and Technology (NIST). 


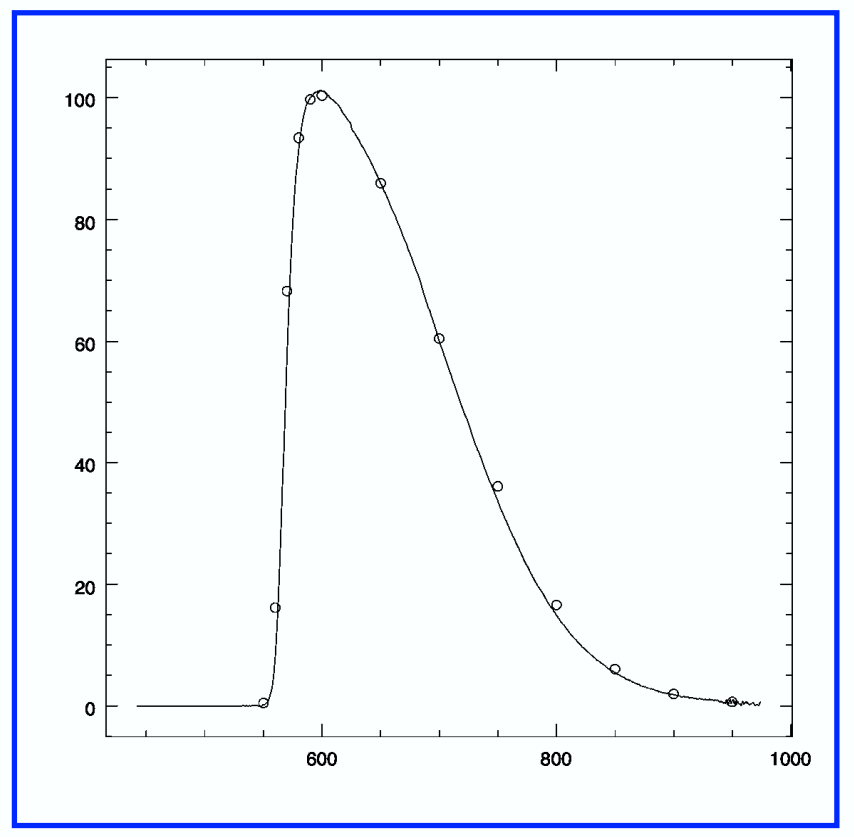

Figure 6: Measurement of the response function of the $r$ band filter on the CT4.0m/MOSAIC telescope at Cerro Tololo. The horizontal axis is wavelength ( $\mathrm{nm})$ and the vertical axis is normalized flux (\%). The open circles are measurements made with a tunable laser illumination system, and the curve is the site standard for the filter. The circles have been normalized to the standard at 700nm. These measurements are stable to better than $1 \%$. 


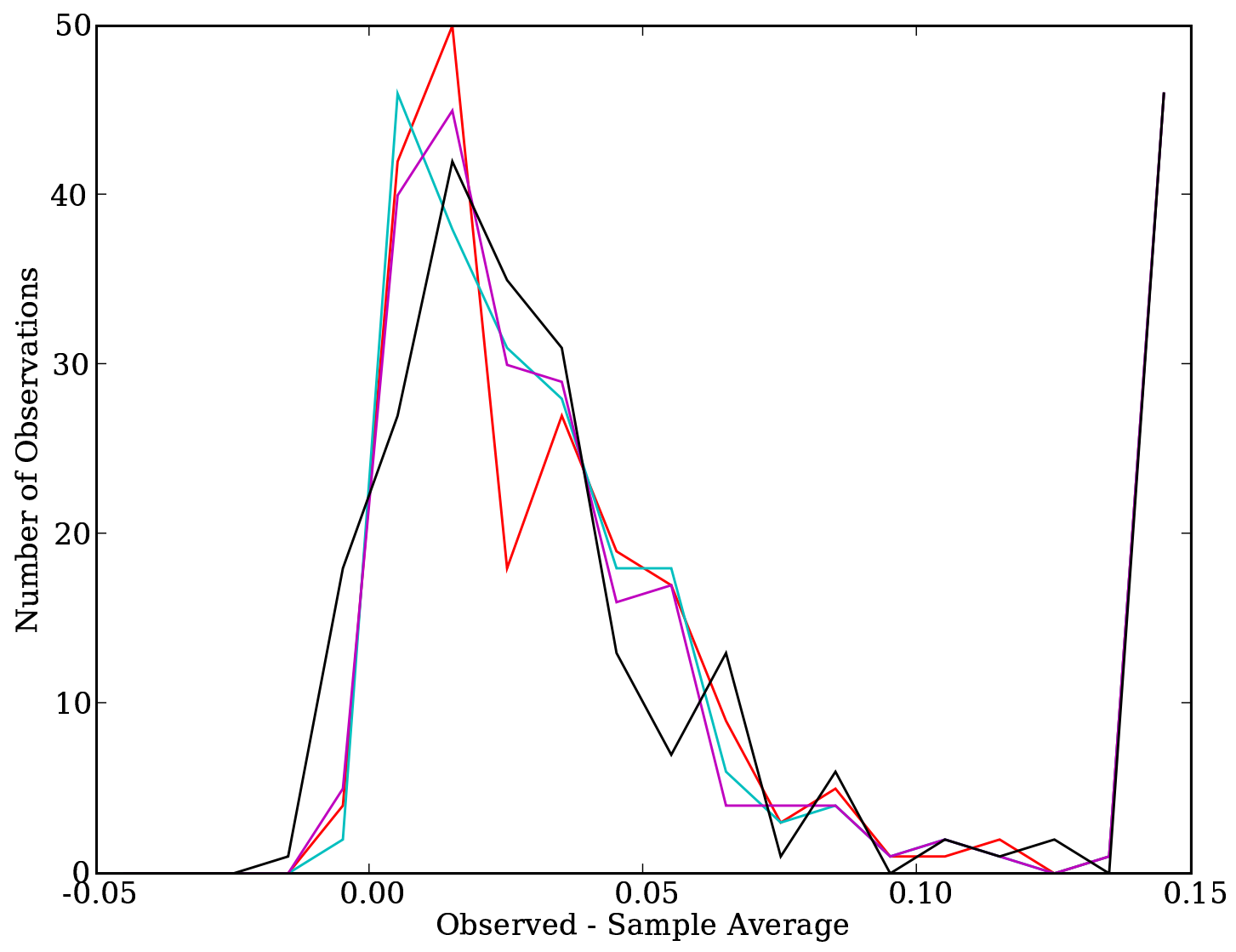

Figure 7: Offsets of blind observations when no "gray" correction is applied. The horizontal axis is the difference between magnitudes of individual observations and the mean of all observations of the same star after correcting for the "gray" absorption. Over and under flow counts are accumulated in bins at the extreme ends of the horizontal range. Values are shown for $r$ (red), $i$ (cyan), $z$ (magenta), and $y$ (black) bandpasses. 


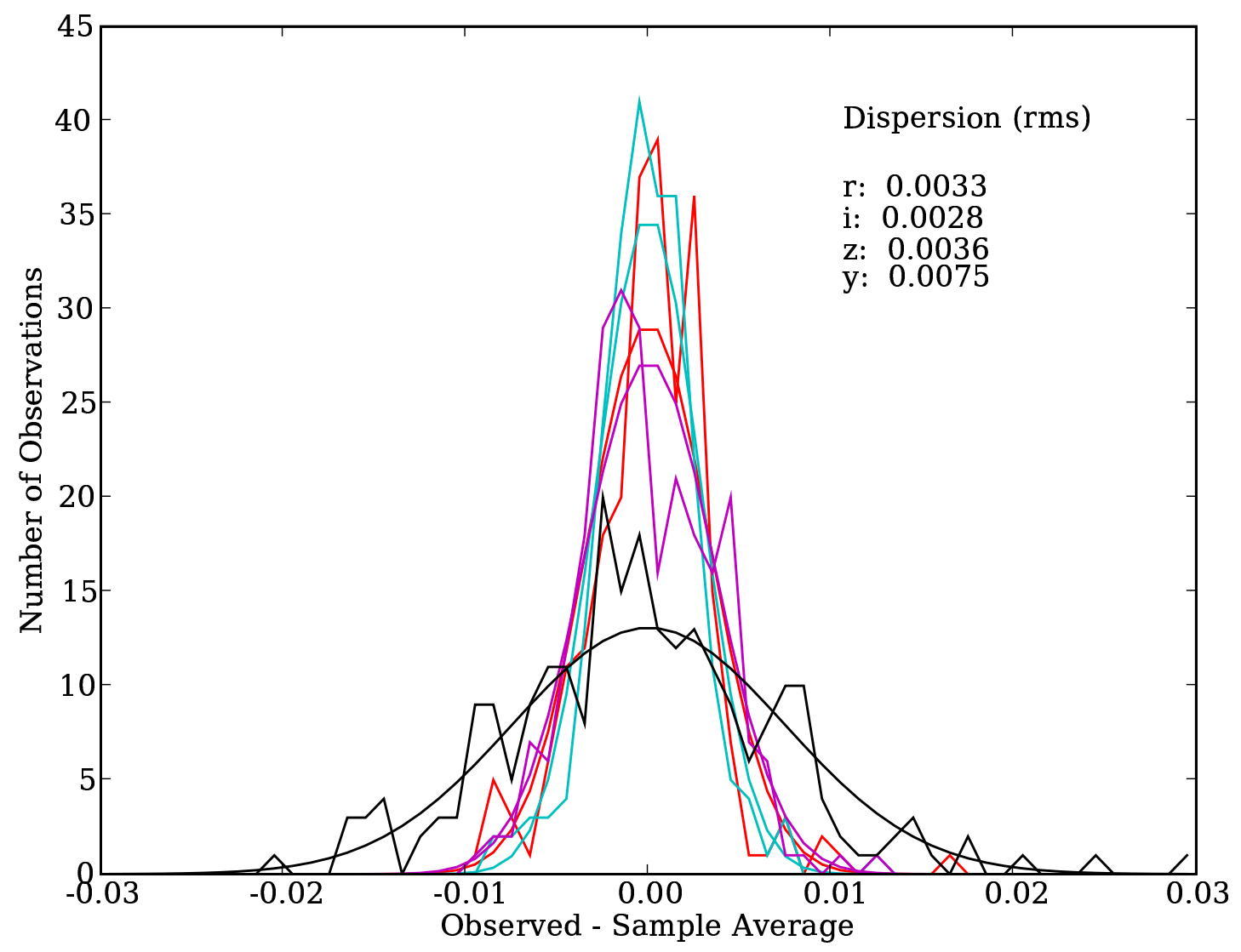

Figure 8: Offsets (dispersion) of blind observations after correction for "gray" extinction fitted to each observation. The horizontal axis is the same as that in Figure 7, but note the different scales. Values are shown for $r$ (red), $i$ (cyan), $z$ (magenta), and $y$ (yellow) bandpasses. The curves are Gaussian functions with $\sigma$ equal to the rms of the offsets of individual observations from their corresponding mean. 


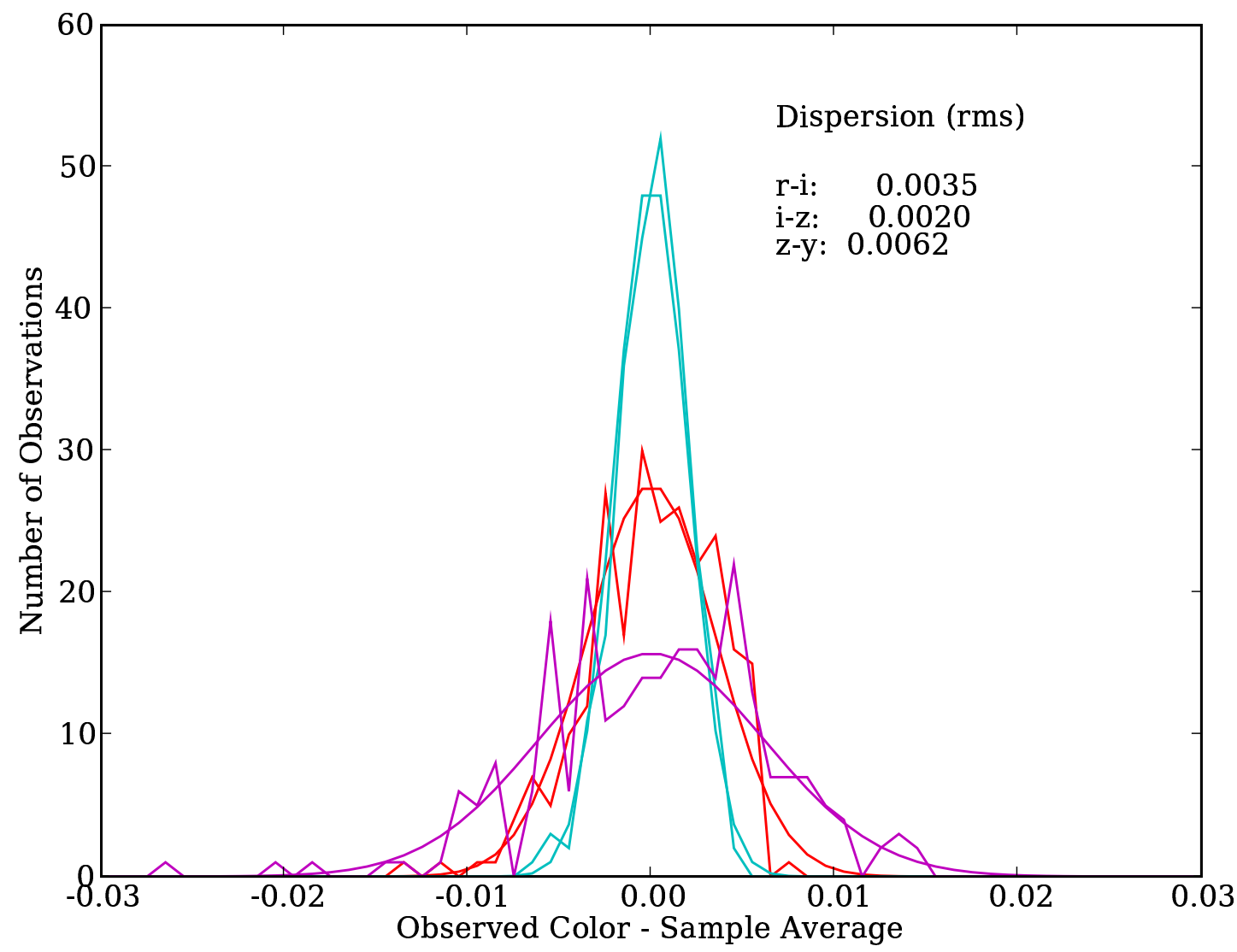

Figure 9: Offsets (dispersion) of colors computed from magnitudes of blind observations. The horizontal axis is the difference between colors of individual blind observations and the mean color of all observations of the same star. Over or under flow counts are accumulated in bins at the extreme ends of the horizontal range. Values are shown for $r-i$ (red), $i-z$ (cyan), and $z-y$ (magenta) colors. The curves are Gaussian functions with $\sigma$ equal to the rms of the offsets of individual observations from their corresponding mean. 


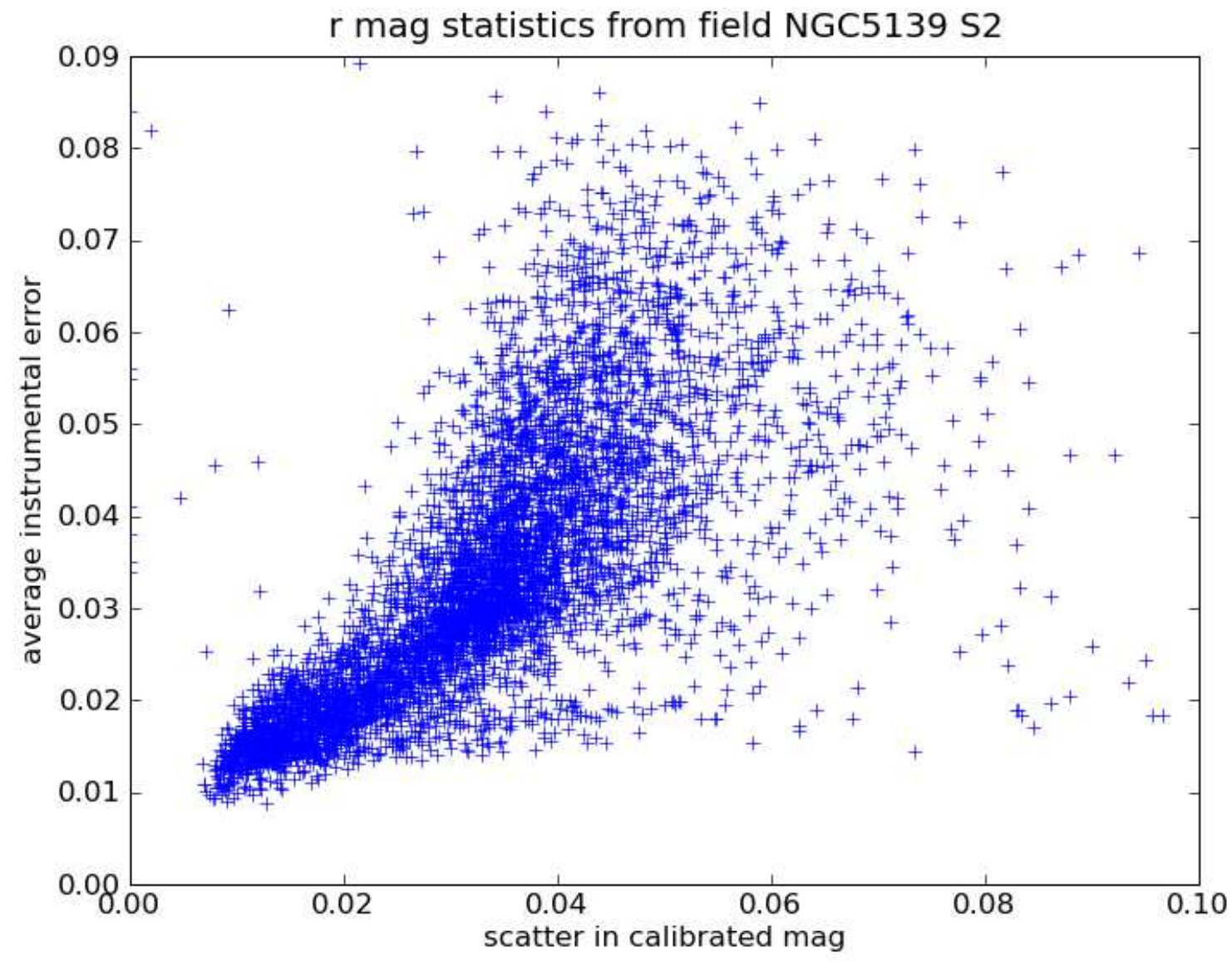

Figure 10: Photometric errors for all matched objects in the field. Each point in the scatter plot represents a particular object's lightcurve, with typically 150 points. The y-axis shows the average instrumental error as reported by dophot, while the $\mathrm{x}$-axis is the observed standard deviation of the lightcurve after correction. Bright objects start at the lower-left and fainter objects range toward upper-right. The band of objects below and well to the right of the $\mathrm{y}=\mathrm{x}$ line are found to be real variable stars. 